\title{
Evaluación de cultivares de caña de azúcar (Saccharum spp.) para producción de panela en el departamento de Boyacá, Colombia
}

\section{Evaluation of sugarcane cultivars (Saccharum spp.) for panela production in the department of Boyacá, Colombia}

Ayda F. Barona-Rodríguez'; Orlando I. Insuasty-Burbano²; Carlos A. Viveros-Valens ${ }^{3}$; Juan C. Ángel-Sánchez ; J Julio Ramírez-Durán ${ }^{5}$

11.ng. Agrónoma, Esp., Estudiante M.Sc. Universidad Pedagógica y Tecnológica de Colombia - UPTC. Corporación Colombiana de Investigación Agropecuaria - Agrosavia, C.I. Tibaitatá, sede Cimpa. Barbosa - Santander, Colombia; e-mail: abarona@agrosavia.co; Dhttps://orcid.org/0000-0001-8487-2727

${ }^{2}$ Ing. Agrónomo. Corporación Colombiana de Investigación Agropecuaria - Agrosavia. Barbosa - Santander, Colombia; e-mail: oribu6755@yahoo.com; (1) https://orcid.org/0000-0001-8844-464X

${ }^{3}$ Ing. Agrónomo, Ph.D. Centro de investigación de la caña de azúcar de Colombia - Cenicaña. Cali, Colombia; e-mail: caviveros@cenicana.org; (D) https:// orcid.org/0000-0001-6824-1722

${ }^{4}$ Ing. Agrónomo, M.Sc. Centro de investigación de la caña de azúcar de Colombia - Cenicaña. Cali, Colombia; e-mail: jcangel@cenicana.org; (D) https://orcid. org/0000-0003-1536-9938

${ }^{5}$ Ing. Agrónomo, M.Sc. Corporación Colombiana de investigación Agropecuaria - Agrosavia, sede Central. Bogotá, Colombia; e-mail: jramirezd@agrosavia.co; (D) https://orcid.org/0000-0002-3385-5748

Cómo citar: Barona-Rodríguez, A.F.; Insuasty-Burbano, O.I.; Viveros-Valens, C.A.; Ángel-Sánchez, J.C.; Ramírez-Durán, J. 2020. Evaluación de cultivares de caña de azúcar (Saccharum spp.) para producción de panela en el departamento de Boyacá, Colombia. Rev. U.D.C.A Act. \& Div. Cient. 23(1):e1298. http://doi.org/10.31910/rudca.v23.n2.2020.1298

Artículo de acceso abierto publicado por Revista U.D.C.A Actualidad \& Divulgación Científica, bajo una licencia Creative Commons CC BY-NC 4.0

Publicación oficial de la Universidad de Ciencias Aplicadas y Ambientales U.D.C.A, Institución de Educación Superior Acreditada de Alta Calidad por el Ministerio de Educación Nacional.

Recibido: Julio 7 de 2019

Aceptado: Julio 4 de 2020

Editado por: Ingeborg Zenner de Polanía

\section{RESUMEN}

En Colombia, el departamento de Boyacá tiene uno de los mayores rendimientos de caña de azúcar (Saccharum spp.), para la producción de panela; sin embargo, los productores demandan constantemente la evaluación de material genético, que pueda aumentar los rendimientos por unidad de área y las características agroindustriales de los materiales tradicionales, de mayor dominio en la región. Con la fi- nalidad de estimar los rendimientos de caña y de panela por hectárea, se evaluaron, a nivel experimental, los cultivares CC 01-1940, CC 99-2282, CC 05-940, CC 03-469, CC 06-791, en el municipio de Moniquirá, Boyacá. Posteriormente, se realizaron pruebas comerciales de molienda, con el mejor cultivar en Moniquirá y en San José de Pare, Boyacá, junto a los testigos de cada zona Caña Buena y RD 75-11, respectivamente. Experimentalmente, se destacó el cultivar CC 01-1940, con una producción de 183,3t/ ha de caña (TCH), 24,2 toneladas de panela por hectárea (TPH), así como una conversión 
de 13,2\% de panela (CP). En prueba comercial de molienda en San José de Pare, se encontró que CC 01-1940 alcanzó una producción de 221,9 TCH y 26,1 TPH, superando por más de 30 toneladas de caña y 5,2 toneladas de panela, al reporte alcanzado por RD 75-11. Para el municipio de Moniquirá, CC 01-1940 obtuvo 158,5 TCH, 18,5 TPH y 11,6\% CP, superando al cultivar testigo Caña Buena.

Palabras clave: Saccharum spp.; Panela; Variedades; Rendimiento; Agroindustria.

\section{ABSTRACT}

In Colombia, the department of Boyacá has one of the highest yields of sugar cane (Saccharum spp.) for panela production. However, producers constantly demand the evaluation of genetic material that could increase the yields per area and the agro-industrial characteristics of the traditional material of greater dominance in the region. In order to estimate cane and panela yields per hectare, cultivars CC 01-1940, CC 99-2282, CC 05-940, CC 03-469, CC 06791 were evaluated experimentally in the municipality of Moniquirá, Boyacá. Subsequently, commercial milling tests were carried out with the best cultivar in Moniquirá and San José de Pare, Boyacá, together with the control materials from each zone, Caña Buena and RD 75-11, respectively. Experimentally, the cultivar CC 01-1940 stood out with a production of $183.3 \mathrm{t} /$ ha of sugarcane (TCH), 24.2 tons of panela per hectare (TPH), as well as a conversion of $13.2 \%$ of panela (CP). In a commercial milling test in San José de Pare, it was found that the cultivar CC 01-1940 had a production of 221.9 TCH and $26.1 \mathrm{TPH}$, surpassing by more than 30 tons of cane and 5.2 tons of panela, for what was reported by RD 75-11. For the municipality of Moniquirá, the cultivar CC 01-1940 obtained 158.5 TCH, 18.5 TPH and 11.6\% CP, surpassing the cultivar Caña Buena.

Keywords: Saccharum spp.; Sugarcane; Varieties; Yield; Agroindustry.

\section{INTRODUCCIÓN}

En Colombia, se cultivan más de 218.749 hectáreas de caña de azúcar, para una producción de panela de 1.391.995 toneladas (Agronet, 2018). Una de las principales zonas de producción del país es la zona geográfica, denominada "Hoya del río Suárez" (HRS), compuesta por 25 municipios, de los departamentos de Boyacá y de Santander. En estos departamentos, se produce el 26,49\% de panela, representando un 18\% del total de área sembrada, a nivel nacional (Agronet, 2018).

En la zona productora de panela de los municipios de Moniquirá y parte de San José de Pare, pertenecientes a la HRS, predomina el cultivar "Caña Buena". Según los productores de la región, esta variedad presenta mejores rendimientos, bajo un esquema de manejo agronómico precario en insumos para el control de plagas, fertilización y manejo de arvenses, frente a otros cultivares tradicionales; sin embargo, el dominio de dicho cultivar ocasiona pérdidas por su susceptibilidad al ataque de plagas y de enfermedades, sumado a ciclos vegetativos largos, que afectan su competitividad.
El cultivar RD 75-11, proveniente de República Dominicana, fue inicialmente introducido al Valle del río Cauca, por el Centro de Investigación de la Caña de Azúcar de Colombia (Cenicaña), mostrando baja adaptación y producción de azúcar para esta zona. Posteriormente, en 1988, se introdujo a la zona panelera de la HRS y dados sus buenos resultados de producción de caña y de panela, se realizó la entrega al sector productivo, en 1996. En el 2016, se encontraba establecida en cerca de 29.891 ha en la HRS, de un total de 42.701 sembradas en el país. Esto representa cerca del 70\% del área total sembrada, a nivel nacional (Murcia \& Ramírez, 2017; Vergara et al. 2018).

El objetivo de la investigación fue evaluar la productividad de nuevos cultivares de caña de azúcar y su desempeño en el proceso agroindustrial, en dos municipios del departamento de Boyacá, en comparación con el comportamiento agroindustrial y fitosanitario de los cultivares RD 75-11 y Caña Buena, ampliamente utilizadas en la región.

\section{MATERIALES Y MÉTODOS}

Cultivares seleccionados: Se seleccionaron cinco genotipos provenientes de Cenicaña, identificados con las siglas CC 01-1940, CC 99-2282, CC 05-940, CC 03-469, CC CC 06-791 y como testigos regionales RD 75-11 y "Caña Buena", ampliamente utilizados en los ambientes de estudio.

Fases de evaluación. La evaluación de los cultivares seleccionados, se desarrolló en dos fases: Una prueba de evaluación agronómica, a nivel de parcela experimental, con mediciones de carácter agronómico e industrial, donde fueron evaluados todos los materiales provenientes de Cenicaña, junto con un testigo regional "Caña buena"; de ahí, fue seleccionado el material con mejor comportamiento para ser evaluado en una segunda fase, pruebas de molienda comercial, comparado con dos testigos regionales, en dos localidades de Boyacá.

Prueba experimental. Se sembraron los genotipos CC 01-1940, CC 99-2282, CC 05-940, CC 03-469, CC 06-791 y la variedad testigo "Caña Buena", en la finca La Esperanza, vereda Ubaza, en el municipio de Moniquirá (altitud de 1608m s.n.m., precipitación promedio entre 2.000 a $2.500 \mathrm{~mm}^{2}$ año $^{-1} \mathrm{y}$ temperatura promedio de $20^{\circ} \mathrm{C}$ ), utilizando un diseño experimental de bloques completos al azar, con cuatro repeticiones. Cada unidad experimental (UE) estuvo constituida por tres surcos de $10 \mathrm{~m}$ de longitud, con una distancia entre surcos de 1,30 para un área útil, por UE, de $39 \mathrm{~m}^{2}$. Simultáneamente, a las labores de siembra, se realizó la toma de la muestra de suelo para su análisis. El plan de fertilización, se realizó de acuerdo con el análisis de suelos y a los requerimientos nutricionales, definidos por Manrique (2000), para el cultivo de la caña de azúcar en la región (100kg de $\mathrm{N}+100 \mathrm{~kg}$ de $\mathrm{P}_{2} \mathrm{O}_{5}+80 \mathrm{~kg}$ de $\mathrm{K}_{2} \mathrm{O}$ por ha).

Evaluación agronómica y de productividad. En la evaluación del desempeño de los materiales genéticos, se consideraron las siguientes variables agronómicas: altura de planta (ALPLA), en 10 tallos tomados al azar por parcela, medida desde la base del tallo hasta la primera hoja con cuello visible; diámetro del tallo (DIATA), 
en 10 tallos tomados al azar por parcela, medido en la parte central del tallo y en la mitad del entrenudo; población de tallos $(\mathrm{P}(\mathrm{t} / \mathrm{m})$ ), conteo de los tallos presentes en $10 \mathrm{~m}$ en el surco central; floración, deshoje natural, comportamiento fitosanitario y datos de producción de caña y de panela, análisis físico químico de jugos y de panela.

Los seguimientos agronómico e industrial periódicos, se realizaron a los 45 días después de la siembra (dds), 3, 6, 9, 12 meses y cosecha. Al momento de la cosecha, se tomaron datos de las siguientes variables: población de tallos molibles, en $10 \mathrm{~m}$ lineales, ALPLA, DIATA; rendimiento de caña por unidad experimental (kg caña por unidad experimental extrapolado a toneladas de caña por ha - TCH) e índice de madurez fisiológico (IDM).

Evaluación de enfermedades. En las inspecciones sanitarias realizadas a los 6 y 9 meses de edad, se empleó la metodología y las escalas descriptivas existentes para la evaluación y la determinación de incidencia y severidad de enfermedades en caña de azúcar (Chavarría, 2006; Ángel et al. 2016; Gabriel et al. 2017). Se hizo énfasis en enfermedades de importancia económica, como roya café, Puccinia melanocephala Syd. and P. Syd, roya naranja, Puccinia kuebnii Butler, virus del mosaico común (SCMV), escaldadura de la hoja, Xanthomonas albilineans (Ashby) Dowson y carbón de la caña de azúcar, Sporisorium scitamineum (Syd.) M. Piepenbr, junto con enfermedades de menor importancia económica, como mancha de ojo, Bipolaris sacchari (E. J. Butler) Shoemaker, mancha de anillo, Leptosphaeria sacchari Breda de Haan, y mancha café, Cercospora longipes Butler.

Molienda fase experimental. Se cosecharon en campo, aproximadamente, $1.500 \mathrm{~kg}$ de caña de cada genotipo y se procesaron en un prototipo de hornilla, tipo Ward Cimpa, como la descrita por Velásquez et al. (2019), con una capacidad de 50kg hora-1 de panela. Para realizar la molienda, se registró el peso de caña entrante a un molino R14, marca "El Panelero" y al final, el peso de panela obtenido, para determinar el TPH y CP (\%), a panela de cada uno de los materiales genéticos.

Caracterización fisicoquímica de jugos y panela. Una vez definido el estado de maduración ideal (relación del valor apical del brix sobre el basal), comprendido entre 0,95 a 1,0 (adimensional), para cada material en evaluación, se procedió a realizar la cosecha y posterior molienda, para la elaboración de panela. Se realizaron por cada cultivar las siguientes acciones: i) cosecha y transporte de caña al sitio de molienda, ii) pesaje de los tallos de caña de azúcar para obtener la TCH, iii) organización y pesado en el patio de apronte, iv) molienda y extracción de jugos, v) obtención y pesaje de panela para obtener TPH y vi) cálculo de la conversión en panela (CP). Dicha metodología, se realizó de la misma manera en la fase experimental y comercial, como lo describe Ramírez et al. (2014a).

Las muestras de jugo y de panela, se enviaron para su proceso y caracterización al laboratorio de fisicoquímica de la sede Cimpa de Agrosavia, para el análisis de las siguientes variables en jugos y panela: $\mathrm{pH}$, sólidos solubles totales ( $\left.{ }^{\circ} \mathrm{Brix}\right)$, sacarosa (\%), azúcares reductores (\%) y humedad (\%); esta variable solo se analizó en panela.
La comparación de cada una de las variables, tanto en jugos como en panela, se hizo con base en los valores de referencia establecidos, de acuerdo con Corpoica (2007) y según los parámetros para la calidad de panela, regidos por la resolución 779/2006, emanada por el Ministerio de la Protección Social en Colombia (Res. 779 del 2006). Estas variables fueron usadas en trabajos previos por López Lopera \& Tamayo Vélez (2017), para el departamento de Antioquia y Bastidas et al. (2012), en Venezuela.

Los resultados, se analizaron mediante el programa estadístico SAS (B) versión 9.0. (SAS, 2004); se realizó Análisis de Varianza (ANAVA) y prueba de comparación de medias (Tukey), entre los tratamientos. Para la elaboración de curvas de isoproductividad, donde se relacionan variables de rendimiento, se usó el sofware ISOS Versión 3.0 (Copyrigh) (ISOS, 2001).

Caracterización de parámetros de calidad en panela. La valoración de la calidad de la panela, se realizó en cuatro variables básicas: aspecto general, textura, brillo y sabor. El aspecto general, se calificó visualmente, en una escala de 1 a $5(1=$ Muy Malo y $5=$ Muy bueno o excelente aspecto). La variable textura, relacionada directamente con la dureza de la panela, se valoró en una escala de 1 a 5 (1= Muy blanda y $5=$ Muy dura). El brillo natural, se calificó con una escala de 1 a 5 ( $1=$ Muy opaca y $5=$ Muy brillante); finalmente, la variable sabor, se calificó con una escala de 1 a $5(1=$ muy salobre y $5=$ muy dulce). Se procedió a calificar la calidad de la panela tomando, como referencia, un puntaje medio de las cuatro variables evaluadas, en las cuales, se determinó si la calidad del producto era: Muy mala, Mala, Regular, Buena y Excelente. El color de la panela, se determinó teniendo como referencia la Tabla Munsell (Munsell, 1946).

Pruebas de molienda comercial. En la prueba de molienda comercial, se usó la variedad con el mejor rendimiento en la prueba experimental, para las variables TCH, TPH y CP y se comparó con RD 75-11 y Caña Buena. Se seleccionaron dos localidades: en San José de Pare (altitud de 1.690m s.n.m., con una precipitación anual entre 1.800 a $2.000 \mathrm{~mm}$ año ${ }^{-1}$ y una temperatura media, que varía entre los 18 a $20^{\circ} \mathrm{C}$ ); se utilizó el cultivar escogido comparado con el testigo regional RD 75-11 y para Moniquirá (altitud de $1.605 \mathrm{~m}$ s.n.m., precipitación anual entre 2.000 a $2.500 \mathrm{~mm}^{2}$ año ${ }^{-1} \mathrm{y}$ temperatura promedio de $20^{\circ} \mathrm{C}$ ), se comparó el cultivar seleccionado con el testigo regional "Caña Buena". En cada localidad, se estableció una parcela de una hectárea con cada uno de los cultivares en evaluación, para la obtención de caña de azúcar, suficiente para la evaluación, a nivel de molienda comercial y producción de panela. Cada uno de los cultivares recibió el mismo modelo de manejo agronómico por parte del agricultor. La fertilización, se realizó previo análisis de suelos y ajustando las cantidades, de acuerdo con las necesidades nutricionales del cultivo (Manrique, 2000). La nutrición fue suministrada en dos fases: un $50 \%$, tres meses después de siembra y el $50 \%$ restante, a los cinco meses de edad.

Evaluación de variables agronómicas. Al momento de la cosecha, se evaluó la producción de caña en campo y en trapiche, registrando la producción de tallos y su rendimiento en panela. En cada cultivar, se evaluaron las variables agronómicas que guardan relación con las 
características de los cultivares para molienda: $\mathrm{P}(\mathrm{t} / \mathrm{m})$; ALPLA (m); DIATA (cm); IDM; TCH; TPH; CP\%.

\section{RESULTADOS Y DISCUSIÓN}

\section{Prueba experimental}

Evaluación agronómica y de productividad, a nivel experimental. La variable $\mathrm{PT} / \mathrm{m}$ no presentó diferencias estadísticas (Tukey $<0,05$ ), con una media de 9,84 tallos/m, valores que concuerdan con los encontrados por Ramírez et al. (2014), en evaluación de cultivares en Santander.

En la variable ALPLA, con la clasificación dada por López Lopera \& Tamayo Vélez (2017), los cultivares CC 01-1940, CC 03-469 y CC 06-791, se clasificaron como tallos de elongación media; al mismo tiempo, presentaron diferencias significativas frente al cultivar CC 99-2282, catalogado como un cultivar de tallos cortos.

Referente al diámetro de los tallos, el cultivar CC 01-1940 presentó diferencias estadísticas frente al cultivar CC 06-791; sin embargo, todos los cultivares evaluados se clasificaron como de grosor medio, con diámetros entre 2,5-3,5cm (López Lopera \& Tamayo Vélez, 2017). Respecto al IDM, su valor varió entre 0,91 y 0,97 , con un promedio de 0,94 , considerado como adecuado para la cosecha; no se presentaron diferencias estadísticas para esta variable (Tabla 1).

En relación con TCH no se observó diferencia estadística entre el cultivar con el valor medio más alto (CC 01-1940) comparado con el testigo regional "Caña Buena"; estos dos materiales obtuvieron los valores mayores y mostraron diferencia estadística frente a los cultivares CC 99-2282 y CC 06-791(Tabla 1). Estos resultados fueron similares a los obtenidos por Briceño et al. (2014), en una evaluación de clones de caña de azúcar, realizada en Venezuela. Para la variable TPH, el cultivar CC 01-1940 mostró diferencias significativas frente a los demás cultivares evaluados, al producir 3,7t de panela adicionales, al ser comparado frente al segundo mejor tratamiento "Caña Buena"; lo anterior, le significaría al productor un ingreso adicional por hectárea, de $\$ 5.457 .500$, teniendo como referencia el precio promedio en Boyacá (Colombia), reportado por Fedepanela, para el periodo 2019

Finalmente, la conversión porcentual en panela $(\mathrm{CP} \%)$, no fue significativo entre los cultivares estudiados y su valor osciló entre 11,9 y $13,2 \%$, con una media del $12,5 \%$ (Tabla 1 ).

La relación de las variables TCH, TPH y rendimiento fue analizada mediante curvas de isoproductividad (Figura 1), donde el cultivar CC 01-1940 mostró los mejores resultados, al ubicarse en el cuadrante superior derecho, que converge una alta producción de caña y un alto valor de conversión a panela.

Comportamiento fitosanitario de las variedades. En la inspección realizada a los seis y nueve meses, los grados de reacción de enfermedades evaluadas (roya café, roya naranja, mancha de anillo y mancha café) fueron inferiores a 3, lo que, de acuerdo con López Lopera \& Tamayo Vélez (2017), se considera bajo y los porcentajes de severidad inferiores al 10\%, de la lámina foliar afectada.

Conviene mencionar que, en el momento en que se realizaron las observaciones del comportamiento fitosanitario de estos materiales genéticos, no se evidenció afección por Escaldadura de la hoja, Raquitismo de las socas y Carbón de la caña, enfermedades de importancia económica.

Tabla 1. Valores promedios de las variables agronómicas evaluadas en cultivares de caña de azúcar, a nivel experimental, en el municipio de Moniquirá, Boyacá.

\begin{tabular}{|c|c|c|c|c|c|c|c|}
\hline Variedad & $\mathbf{P}(\mathrm{t} / \mathrm{m})$ & ALPLA (m) & DIATA $(\mathrm{cm})$ & IDM & TCH & TPH & $\mathrm{CP} \%$ \\
\hline CC 01-1940 & $9,73 \mathrm{a}$ & $3,01 \mathrm{a}$ & $3,31 \mathrm{a}$ & $0,97 \mathrm{a}$ & $183,3 \mathrm{a}$ & $24,2 \mathrm{a}$ & $13,2 \mathrm{a}$ \\
\hline CC 99-2282 & $10,50 \mathrm{a}$ & $2,40 \mathrm{~b}$ & $3,12 \mathrm{ab}$ & $0,93 \mathrm{a}$ & $135,9 \mathrm{c}$ & $16,2 \mathrm{c}$ & $11,9 \mathrm{a}$ \\
\hline CC 05-940 & $9,80 \mathrm{a}$ & $2,85 \mathrm{ab}$ & $3,09 \mathrm{ab}$ & $0,95 \mathrm{a}$ & $147,0 \mathrm{bc}$ & $18,2 \mathrm{bc}$ & $12,4 a$ \\
\hline CC 03-469 & $9,70 \mathrm{a}$ & $2,93 \mathrm{a}$ & $3,28 \mathrm{ab}$ & $0,93 \mathrm{a}$ & $158,6 \mathrm{abc}$ & $20,1 \mathrm{~b}$ & $12,7 \mathrm{a}$ \\
\hline CC 06-791 & $9,95 \mathrm{a}$ & $2,91 \mathrm{a}$ & $3,00 \mathrm{~b}$ & $0,95 \mathrm{a}$ & $138,9 \mathrm{c}$ & $17,5 \mathrm{bc}$ & $12,5 \mathrm{a}$ \\
\hline CAÑA BUENA & $9,40 \mathrm{a}$ & $2,89 \mathrm{ab}$ & $3,24 \mathrm{ab}$ & $0,91 \mathrm{a}$ & $169,2 \mathrm{ab}$ & $20,7 \mathrm{~b}$ & $12,3 \mathrm{a}$ \\
\hline Media & 9,84 & 2,83 & 3,17 & 0,94 & 155,5 & 19,5 & 12,5 \\
\hline Tuckey & $\mathrm{X}$ & 0,51 & 0,29 & $\mathrm{x}$ & 25,4 & 3,5 & $\mathrm{X}$ \\
\hline Significancia & ns & 0,05 & 0,05 & ns & 0,05 & 0,05 & ns \\
\hline
\end{tabular}

P (t/m): Población de tallos por metro; ALPLA: Altura de planta (m); DIATA: Diámetro del tallo (cm); IDM: Índice de madurez fisiológico; TCH: Toneladas de caña por hectárea; TPH: Toneladas de panela por hectárea; CP\%: Porcentaje de conversión en panela. Valores seguidos con la misma letra no son significativamente diferentes $(\boldsymbol{a}=0,05)$ para la prueba de Tukey. ns: No significativo. Valores seguidos con letra diferente son significativamente diferentes $(\boldsymbol{a}=0,05)$ para la prueba de Tukey. 


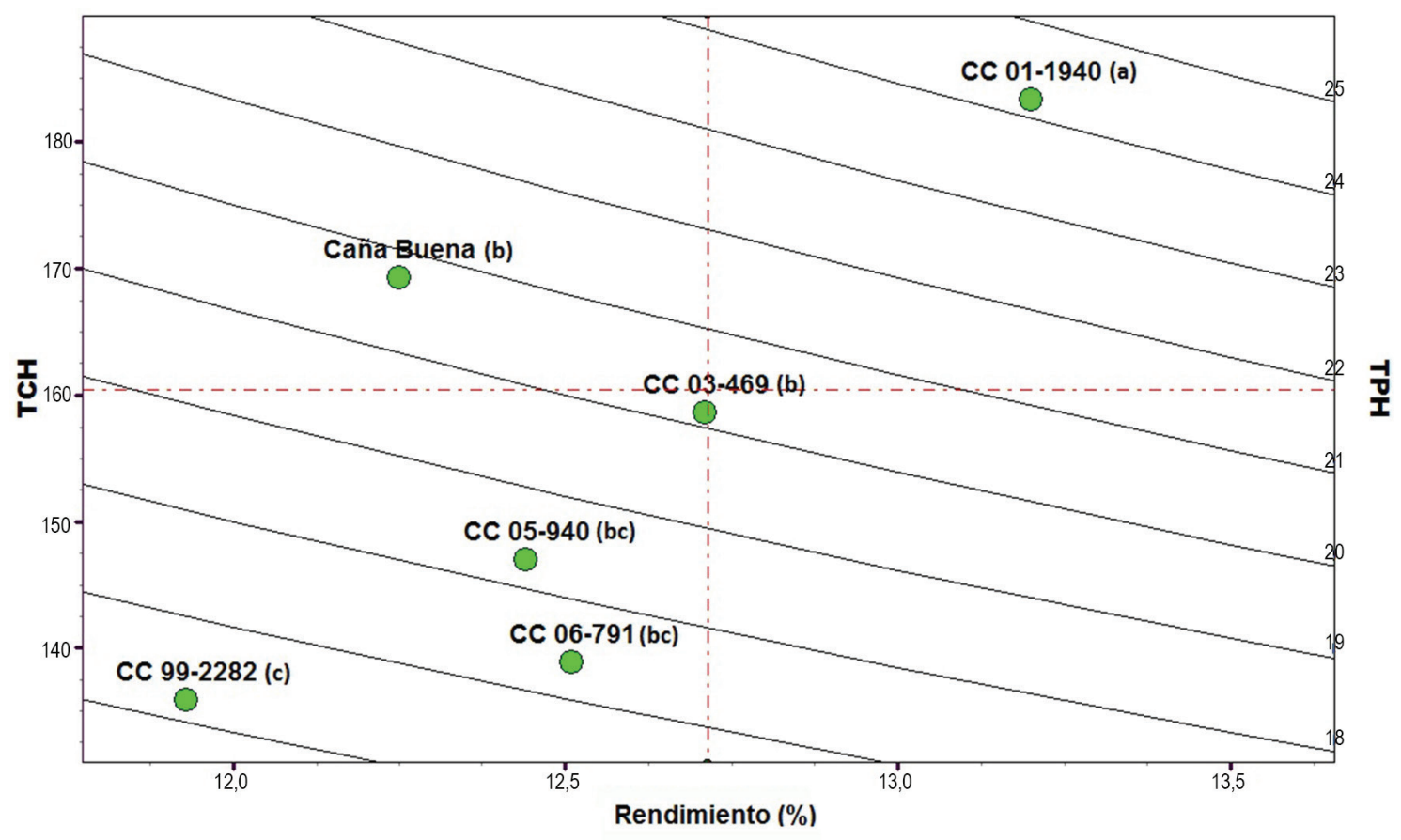

Figura 1. Curvas de isoproductividad en seis variedades de caña para producción de panela a nivel experimental en Moniquirá, Boyacá

Caracterización fisicoquímica de jugos y panela. La caracterización fisicoquímica de los jugos indicó que, con relación a la variable $\mathrm{SST} \%$, los seis materiales evaluados estuvieron dentro del promedio referenciado como adecuado, entre 16 al 24\% (López Lopera \& Tamayo Vélez, 2017). En cuanto al pH, los valores encontrados fluctuaron entre 5,4 y 5,5, es decir, entre el rango establecido por Corpoica (2007), para obtener panela con buenas características comerciales. Con relación al contenido de azúcares reductores, los resultados obtenidos fluctuaron entre 0,6 y 1,5\%. Un valor del $2 \%$ de contenido de azúcares reductores en jugos se considera alto y puede afectar la calidad de los jugos por cuanto induce a una mayor concentración de glucosa y de fructosa (Durán, 2010).

El contenido de sacarosa (\%) está relacionada con una buena textura de la panela; los valores obtenidos oscilaron entre 18,6 a 20\%, apropiados, ya que, de acuerdo con Ramírez et al. (2014a), contenidos inferiores al 18\% se consideran bajos. Finalmente, el valor de la pureza varió entre 92,1 a 97,1\%, siendo la variedad CC 01-1940 la de mejor tendencia, por pureza en jugos.

Para la caracterización fisicoquímica de la panela, los análisis indicaron, para azúcares reductores, valores entre 7,04 y 10,64\%, acordes con la resolución 779 de 2006, que determina como valor mínimo requerido para una panela de buena calidad, el 5,5\%. El contenido de azúcares reductores altos en panela afecta directamente su textura, volviéndolas melcochudas y de alta perecibilidad, ya que concentran mayores niveles de glucosa y de fructosa (Durán, 2010). De acuerdo con la misma resolución, se destaca el comportamiento de las panelas para la variable sacarosa, las cuales, están por debajo de $83 \%$, valor permitido como máximo (MPS - Resolución 779 del 2006). De acuerdo con Ramírez et al. (2014a), los cultivares desarrollados por Cenicaña presentan altos valores en esta variable, dado que son seleccionados, en primera instancia, como promisorios, para la producción de azúcar.

Finalmente, con relación al porcentaje de humedad en panela, los valores variaron entre 6,8 a 8,1\%, que están dentro del valor máximo permitido del 9\%, estipulado por la Resolución 779/2006.

Caracterización sensorial de calidad en panela. Las panelas, se destacaron por presentar muy buena textura (dura), con un brillo normal; en cuanto al sabor de la panela, las variedades CC 06-791 y Caña buena, se caracterizaron por presentar un sabor muy dulce, a diferencia del resto de materiales que presentaron un sabor dulce normal. Desde el punto de vista de calidad, con base en los parámetros anteriores, la variedad CC 06-791 presentó una panela de muy buena o excelente calidad; el resto de cultivares presentaron una panela de buena calidad y potencialmente competitivas en el mercado. Desde el punto de vista de la valoración del color, las panelas obtenidas con las seis variedades presentaron panelas de colores claros (rubias), de acuerdo con la escala de clasificación de la tabla internacional, para determinación del color de Munsell (Munsell, 1946); los colores de las panelas obtenidos con estas variedades son apetecidos, en los diferentes mercados donde se comercializan; en general, los resultados indicaron que la calidad del producto final fue Muy buena o excelente, en los seis materiales (Tabla 2).

\section{Pruebas de molienda comercial}

En el Municipio de San José de Pare, los cultivares alcanzaron su maduración 17 meses después de la siembra, con un IDM de 0,94, para CC 01-1940 y 0,95, para RD 75-11. En cuanto al comporta- 
Tabla 2. Resultados de la evaluación de la calidad de panela de seis variedades de caña de azúcar, a nivel experimental, en el municipio de Moniquirá.

\begin{tabular}{|l|l|l|l|l|l|l|}
\hline Variedad & $\begin{array}{l}\text { Aspecto } \\
\text { general }\end{array}$ & $\begin{array}{l}\text { Calidad } \\
\text { de dureza }\end{array}$ & Brillo & Color & Sabor & $\begin{array}{l}\text { Calidad } \\
\text { final, } \\
\text { Calificación }\end{array}$ \\
\hline CC 01-1940 & Muy bueno & Dura & Brillante normal & 10 YR 5/8 Yellowish brown & Dulce normal & Buena, 4.25 \\
\hline CC 99-2282 & Bueno & Dura & Brillante normal & 10 YR 5/6 Yellowish brown & Dulce normal & Buena, 4.00 \\
\hline CC 05-940 & Bueno & Dura & Brillante normal & 10 YR 5/8 Yellowish brown & Dulce normal & Buena, 4.00 \\
\hline CC 03-469 & Bueno & Dura & Brillante normal & $\begin{array}{l}10 \text { YR 4/6 Dark yellowish } \\
\text { brown }\end{array}$ & Dulce normal & Buena, 4.00 \\
\hline CC 06-791 & Muy bueno & Dura & Brillante normal & 10 YR 5/8 Yellowish brown & Dulce normal & Buena 4.25 \\
\hline Caña buena & Bueno & Dura & Brillante normal & 10 YR 5/6 Yellowish brown & Dulce normal & Buena 4.00 \\
\hline
\end{tabular}

miento agronómico, se observó que CC 01-1940 presentó mayor ALPLA comparada con RD 75-11 (Tabla 3); en esta etapa de la evaluación, el cultivar CC 01-1940 presentó un ALPLA superior a los $3,5 \mathrm{~m}$, por lo cual, se clasifica como de tallos largos, a diferencia de RD 75-11 que, para esta etapa, presentó tallos de longitud media (Ramírez et al. 2014). Respecto al DIATA, los dos cultivares se consideran materiales de tallos medianamente gruesos, comprendidos entre los 2,51 a 3,50 cm (López Lopera \& Tamayo Vélez, 2017) y con relación a la población de tallos $/ \mathrm{m}$, los cultivares obtuvieron entre 8 a 14 tallos $/ \mathrm{m}$, considerándose como materiales de mediana población de tallos (Ramírez et al. 2014). Durante la molienda comercial, se encontró que CC 01-1940 superó por más de 30t de caña y 5,2t de panela al rendimiento alcanzado por RD 75-11. Adicionalmente, el porcentaje de conversión a panela también fue superior por parte del cultivar CC 01-1940, lo que le permite recuperar 117,6kg de panela por $\mathrm{t}$ de caña procesada, en comparación con el de RD 75-11, que recuperaría 109,6kg (Tabla 3).

Al comparar las variables agronómicas de CC 01-194 con "Caña Buena”, en Moniquirá, se encontró, en general, un comportamiento similar de ambos cultivares, clasificándo su $\mathrm{P}(\mathrm{t} / \mathrm{m})$ como mediana, entre 8 a 14 tallos/m; el ALPLA, como de longitud corta y el DIATA, como tallos de tamaño grueso, comprendidos entre los 2,51 a 3,5cm (López Lopera \& Tamayo Vélez, 2017). Pese a esta similitud en estas variables, el cultivar CC 01-1940 presentó un TCH mayor, superando por 7t, el comportamiento de "Caña Buena". De igual manera, CC 01-1940 superó a este cultivar, en 2,6 TPH, influenciado, principalmente, por un alto valor de conversión a panela (Tabla 3).

Las curvas de isoproductividad para las parcelas comerciales (Figura 2), muestra que en las dos localidades, la producción de CC 01-1940 fue la de mejor respuesta en comparación con los testigos, ubicándose en el cuadrante superior derecho, con altos TCH, TPH y rendimiento en panela.

Caracterización fisicoquímica de jugos y panela. Los resultados indicaron, que para los dos cultivares evaluados comercialmente en el municipio de San José de Pare, se observó que el contenido de SST $\%$ en jugos del cultivar CC 01-1940 superó en 1,68\% al obtenido por el cultivar RD 75-11. El pH de los jugos en los dos cultivares mostró un valor adecuado requerido para el proceso de obtención de panela, mayor a 5,5. Respecto al contenido de sacarosa (\%), el cultivar CC $01-1940$ superó en 1,4\% al obtenido por el cultivar RD 75-11. El contenido de AR\%, en los dos cultivares, reportó un valor bajo, inferior al 0,33\%. En términos de pureza en

Tabla 3. Evaluación de variables agronómicas para molienda a nivel comercial de los cultivares de caña de azúcar para producción de panela en las localidades de San José de Pare y Moniquirá, Boyacá.

\begin{tabular}{|l|l|l|l|l|l|l|l|l|}
\hline Cultivar & Localidad & $\mathrm{P}(\mathrm{t} / \mathrm{m})$ & ALPLA & DIATA & IDM & TCH & TPH & CP $\%$ \\
\hline CC 01-1940 & San José de Pare & 10,5 & 3,64 & 3,36 & $0,94 \mathrm{a}$ & 221,9 & 26,1 & 11,76 \\
\hline RD 75-11 & San José de Pare & 10,2 & 3,29 & 3,20 & $0,95 \mathrm{a}$ & 191,0 & 20,9 & 10,96 \\
\hline CC 01-1940 & Moniquirá & 10,0 & 2,83 & 3,38 & $0,97 \mathrm{a}$ & 158,5 & 18,5 & 11,6 \\
\hline Caña Buena & Moniquirá & 10,7 & 2,84 & 3,40 & $0,93 \mathrm{a}$ & 151,5 & 15,9 & 10,5 \\
\hline
\end{tabular}

PT/m: Población de tallos por metro; ALPLA: Altura de planta (m); DIATA: Diámetro del tallo (cm); IDM: Índice de madurez fisiológico; TCH: Toneladas de caña por hectárea; TPH: Toneladas de panela por hectárea; $\mathrm{CP} \%$ : Porcentaje de conversión en panela. 
jugos, el cultivar RD 75-11 superó en 1,3\% al cultivar CC 01-1940 (Tabla 4). En general, la composición fisicoquímica de los jugos de estos dos cultivares permitió la obtención de un producto final de buena calidad (Corpoica, 2007).

La composición fisicoquímica de la panela obtenida con estos dos cultivares, se observó que el contenido de SST\% de CC 01-1940 superó en 1,5\% el valor obtenido por RD 75-11. De igual manera, el cultivar CC 01-1940 superó en 1,9\% a RD 75-11, en el conteni- do de sacarosa (Tabla 4), valores que están dentro de los rangos permitidos por la resolución 779 de 2006, del MPS.

Para la localidad de Moniquirá, el cultivar CC 01-1940 alcanzó en jugos una pureza, a nivel comercial, del $98,2 \%$, como un buen indicador en la relación del contenido de SST\% y su concentración de sacarosa. El cultivar "Caña Buena" tuvo una pureza comercialmente del $96,9 \%$, ligeramente por debajo de los valores alcanzados por CC 01-1940 (Tabla 4).

Tabla 4. Análisis fisicoquímico en jugos y panela, prueba de molienda comercial de cultivares de caña de azúcar.

\begin{tabular}{|l|l|l|l|l|l|l|l|l|}
\hline Variedad & Localidad & Muestra & SST (\%) & $\mathrm{pH}$ & Sacarosa (\%) & AR (\%) & Pureza (\%) & Humedad (\%) \\
\hline CC 01-1940 & San José de Pare & Jugo & 21,53 & 5,6 & 20,6 & 0,27 & 95,6 & N/A \\
\hline RD 75-11 & San José de Pare & Jugo & 19,85 & 5,5 & 19,2 & 0,33 & 96,9 & N/A \\
\hline CC 01-1940 & San José de Pare & Panela & 90,7 & 5,8 & 80,6 & 3,76 & 88,8 & 9,2 \\
\hline RD 75-11 & San José de Pare & Panela & 89,2 & 5,7 & 78,7 & 4,28 & 88,3 & 10,4 \\
\hline CC 01-1940 & Moniquirá & Jugo & 21,8 & 5,6 & 21,4 & 0,50 & 98,2 & N/A \\
\hline Caña Buena & Moniquirá & Jugo & 22,7 & 5,5 & 22,0 & 0,80 & 96,9 & N/A \\
\hline CC 01-1940 & Moniquirá & Panela & 93,2 & 5,9 & 73,5 & 12,60 & 78,9 & 6,8 \\
\hline Caña Buena & Moniquirá & Panela & 92,8 & 6,5 & 77,9 & 8,60 & 84,0 & 7,2 \\
\hline
\end{tabular}

Los resultados del análisis físico químico en panelas indican que ninguno de los cultivares superó el 83,0\% del contenido máximo permitido, para la variable sacarosa (MPS, 2006); de igual manera, el contenido de azúcares reductores en panela estuvo por encima del $5,5 \%$ y la humedad estuvo por debajo del 9,0\% (Tabla 4).

Calidad sensorial de panela. La valoración organoléptica realizada a las panelas obtenidas con los cultivares en estudio fueron de aspecto general muy bueno, con buena textura y de colores claros, características que les da muy buena aceptabilidad en el mercado.

Se determinó que la CC 01-1940 presentó características agronómicas deseables, para las variables ALPLA, DIATA y $\mathrm{P}(\mathrm{t} / \mathrm{m})$. En las dos localidades, la producción fue la de mejor respuesta en comparación con el testigo. A nivel de calidad de panela, también fue superior. Con relación al TCH, TPH y RP\% fue sobresaliente la variedad CC 01-1940, con 183,3; TCH, 24,2; TPH y RP de 13,2\%, superando al testigo "Caña Buena", a nivel experimental.

La valoración fisicoquímica de las panelas de las seis variedades, como contenido de sacarosa (\%), azúcares reductores (\%) y humedad (\%), estuvieron dentro de los rangos permitidos por la Resolución 779/2006, del Ministerio de la Protección Social de Colombia.

En cuanto a la calidad general de la panela relacionada con su aspecto general, brillo natural, sabor, textura y color, como producto terminado, las panelas de las seis variedades evaluadas, incluyendo la variedad testigo, presentaron "buena calidad", de acuerdo con los requerimientos exigidos por los consumidores en el mercado.

A nivel comercial, se presentaron ligeras diferencias entre las localidades; en ambas zonas, se destacó la variedad CC 01-1940, por su comportamiento agronómico, rendimiento, calidad de los jugos y panela, lo que le permite perfilarse como un material promisorio para las zonas paneleras.

Agradecimientos: A la Corporación Colombiana de Investigación Agropecuaria, al Centro de Investigación de la Caña de Azúcar, a los productores Hernán Garavito y Saúl Alberto Mora, por su colaboración y disposición para la ejecución de la propuesta de investigación. Al asistente de investigación Javier Jiménez Vargas, integrante importante del equipo de trabajo, por su apoyo y aportes al trabajo. Conflictos de intereses. El artículo fue preparado y revisado con la participación de todos los autores, quienes declaramos que no existe conflicto de intereses que ponga en riesgo la validez de los resultados presentados. Financiación: Este estudio fue financiado con recursos públicos asignados a las Corporación Colombiana de Investigación Agropecuaria, mediante el macroproyecto titulado "Generación de recomendaciones agronómicas y nuevas tecnologías en la producción de panela en Colombia". 


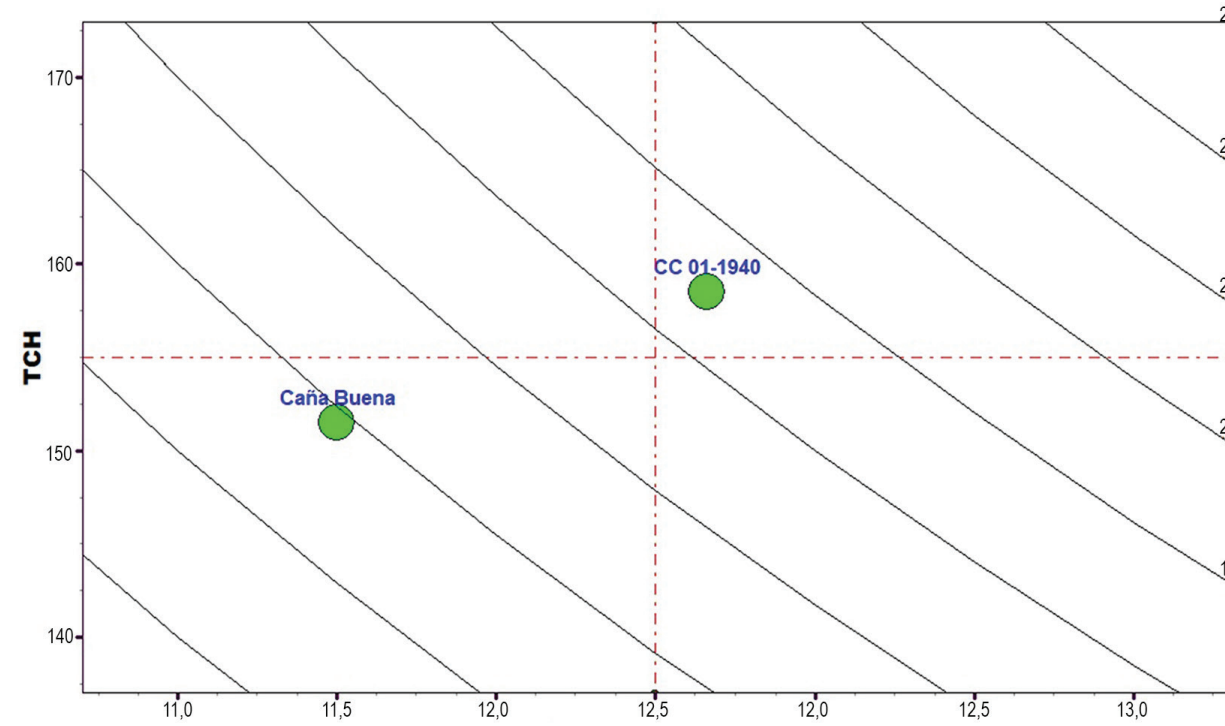

(a)

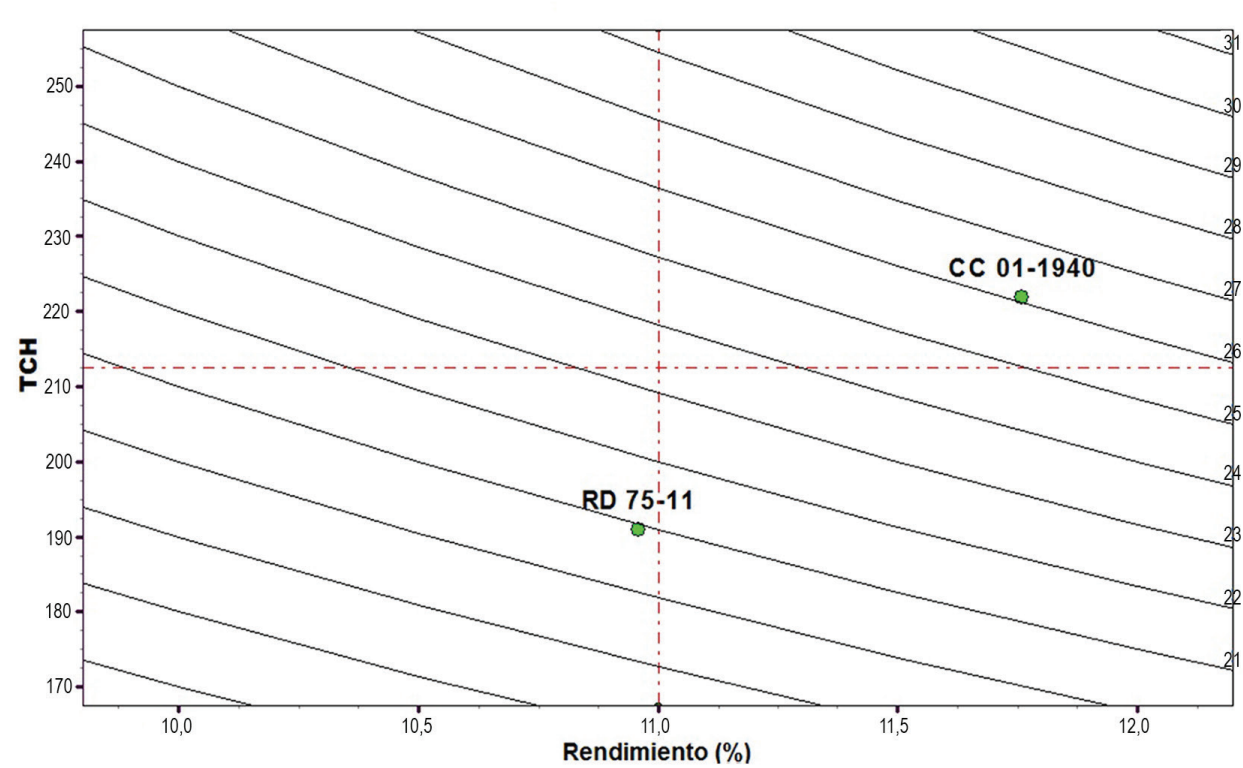

(b)

Figura 2. Curvas de isoproductividad de los cultivares de caña de azúcar para producción de panela en Boyacá: a. Parcela comercial Moniquirá; b. Parcela comercial San José de Pare.

\section{REFERENCIAS}

1. AGRONET. 2018. Estadísticas Agropecuarias. Cultivo de caña panelera. Colombia. Disponible desde Internet en http://www.agronet.gov.co/estadistica/ (con acceso el 03/01/2020).

2. ÁNGEL, J.C.; VICTORIA, J.I.; CADAVID, M.; ÁNGEL, C.A. 2016. The race is on review, advances and challenges for integrated and sustainable management of sugarcane brown rust and orange rust in Colombia. Proc. Internal Soc. of Sugar Cane Technologists. 29:1776-1785.
3. BASTIDAS, L.; REA, R.; DE SOUSA-VEIRA, O.; HERNANDEZ, E.; BRICEÑO, R. 2012. Análisis de variables agronómicas en cultivares de caña de azúcar con fines azucareros, paneleros y forrajeros. Bioagro. 24(2):135-142.

4. BRICEÑO, R.; DE SOUSA, O.; DÍAZ, A.; REA, R.; NIÑO, M.; RIVERO, A.; AZA, G.; GEORGE, J. 2014. Evaluación de clones experimentales de caña de azúcar en etapas tempranas del programa venezolano para el desarrollo de variedades. Agronomía Trop. 64(3-4):165-172.

5. CHAVARRÍA, E. 2006. Escalas descriptivas para la evaluación de enfermedades de la caña de azúcar. LAICA (Costa Rica). 46p. 
6. Corporación Colombiana de Investigación Agropecuaria CORPOICA. 2007. Guía tecnológica para el manejo integral del sistema productivo de la caña panelera. Ed. Produmedios (Bogotá DC). 152p.

7. DURÁN CASTRO, N. 2010. Reingeniería panelera. Búhos Editores (Tunja, Colombia). 244p.

8. Federación Nacional de Productores de Panela - FEDEPANELA. 2019. Sistema de información panelero - SIPA. Boletín semanal de precios. Colombia. Disponible desde Internet en:

http://www.sipa.org.co/wp/index.php/2019/03/06/ precios-historicos/ (con acceso el 14/04/2020).

9. GABRIEL, J.; ORTUÑO, N.; VERA, M.; CASTRO, C.; NARVÁEZ, W; MANOBANDA, M. 2017. Manual para evaluación de daños de enfermedades en cultivos agrícolas. Grupo COMPAS, Universidad Estatal del Sur de Manabí (Jipijapa, Ecuador). 53p.

10. ISOS. 2001. Gráficas ISO, Versión 3, Copyright. Cenicaña. Centro de investigación de la caña de azúcar de Colombia.

11. LÓPEZ LOPERA, J.G.; TAMAYO VÉLEZ, A. 2017. Agroindustrial performance of sugarcane varieties for panela in Antioquia, Colombia. Rev. Facultad Nacional de Agronomía Medellín. (Colombia). 70(3):8303-8310. https://doi.org/10.15446/rfna.v70n3.66329

12. MANRIQUE, R. 2000. Suelos, Nutrición y Fertilización de la caña para panela. En: Parra, O.; Paez, H. (eds). Manual de caña de azúcar para producción de panela. Corpoica Fedepanela. Bucaramanga. Bucaramanga. p.89-96.

13. Ministerio de la protección social en Colombia -MPS. 2006. Parámetros establecidos en calidad de panela. Resolución 779 del 2006. 6p.
14. MUNSELL, A.H. 1946. A Color Notation: An illustrated system defining all colors and their relations by measured scales of hue, value and chrome (15th ed.). Baltimore, Maryland: Munscell Color Co.

15. MURCIA, M.L.; RAMÍREZ, J. 2017. Reconversión del sistema regional de producción de caña para la agroindustria panelera en Boyacá y Santander. Corpoica Ciencia Tecnología Agropecuaria. 18(1):75-87.

http://dx.doi.org/10.21930/rcta.vol18_num1_art:559

16. RAMÍREZ, J.; INSUASTY, O.I.; MURCIA, M.L. 2014. Variedades de caña de azúcar empleadas para la agroindustria panelera de Colombia. Corpoica (Barbosa, Colombia). 116p.

17. RAMÍREZ, J.; INSUASTY, O.I.; VIVEROS, C. 2014a. Comportamiento agroindustrial de diez variedades de caña de azúcar para producción de panela en Santander, Colombia. Corpoica Ciencia Tecnología Agropecuaria. (Colombia). 15(2):183-195.

https://doi.org/10.21930/rcta.vol15_num2_art:358

18. SAS Institute Inc. 2004. Base SAS ${ }^{\circledR} 9.0$ Procedures Guide. Cary, NC: SAS Institute Inc.

19. VELÁSQUEZ, F.; ESPITIA, J.; MENDIETA, O.; ESCOBAR, S.; RODRÍGUEZ, J. 2019. Non-centrifugal cane sugar processing: A review on recent advances and the influence of process on qualities attrbutes of final products. J. Food Engineering. 255:32-40. https://doi.org/10.1016/j.jfoodeng.2019.03.009

20. VERGARA, R.; RODRÍGUEZ, L.; ALARCON, Y. 2018. Estudio de caracterización de la actividad productiva del sector de la caña panelera en la hoya del rio Suárez. NOVUM (Colombia). 1(8):32-48. 